\title{
Depth Analysis of Channel Equalization Algorithm
}

\author{
Anyi Wang, Yuan Sun \\ Xi'an University of Science and Technology, Xi'an, China \\ Xi'an University of Science and Technology, Xi'an, China \\ wanganyi@xust.edu.cn, 550931680@qq.com
}

\begin{abstract}
Keywords: Training Sequence, Non-blind Equalization, ZF Algorithm, MMSE Algorithm, MATLAB.
\end{abstract}
\begin{abstract}
The channel equalization technology is one of the key technologies to improve the quality of communication. It has a vital role to improve overall system performance. In order to overcome the inter symbol interference caused by the channel characteristics of the time-varying and multipath propagation, then make sure that the received signal is correct, communication system must use the channel equalization technology. By sending a bunch of training sequence which was known to the sender and receiver, it can estimate the channel's characteristics, continuously adjust and determine the equalizer's tap coefficients. Then the inter-symbol interference is overcome. So the performance of the system can be improved. In this paper, firstly, the depth analysis of the zero forcing (ZF) algorithm and minimum mean square error (MMSE) algorithm of channel equalization are performed, and their advantages and disadvantages on the theory are summarized. Secondly, simulating of the two algorithms is based on the MATLAB platform, and their advantages and disadvantages by comparing the simulation results are exhibited.
\end{abstract}

\section{Introduction}

The channel of wireless communication is unknown and time-varying. The unknown problem can be solved by sending a training sequence, which is a known bit sequence, but the time-varying problem needs to use the equalization technology. This channel may lead to multipath effect, which could cause inter symbol interference (ISI). It seriously affects the transmission of digital signals. Although the coding and the diversity technology can reduce the error caused by ISI, the error can't be eliminated completely. In order to solve this problem, it's necessary to introduce the equalizer in the receiver to reduce or eliminate inter symbol interference.

The criteria of channel equalization algorithm commonly used are, Zero-Forcing (ZF), Minimum Mean Square Error (MMSE), Least Square (LS), Least Mean Square (LMS), RLS and so on[1][2]. The ZF algorithm was put forward by Lucky in 1965, he omitted the channel's additive noise in his analysis, so this algorithm is not necessarily the best, but easy to implement. In [3], Yongsheng Chen verified that the ZF algorithm can suppress the inter symbol interference in a certain extent in indoor visible light communication system. And in [4], Xuefei Ma put forward the application of MMSE in underwater acoustic channel, the results show that the MMSE equalization algorithm can overcome inter symbol interference, and improve the performance of the system. Thus, the equalization technology is an indispensable part in wireless transmission system, and it is necessary to make in-depth analysis of the equalization technology.

\section{Channel equalization}

This paper adopts non-blind equalization. Its equalizer working process can be divided into two modes, training mode and data transmission mode [5].In training mode, by sending a bunch of bit sequence (training sequence) $C_{i}$, which was known by the sender and receiver, equalizer compares the received blurred signal with the original signal, analyzes and estimates the characteristics of the channel. Then it adjusts its parameters $W_{i}$ constantly, and achieves the purpose that the distortion of 
the received signal is compensated in the equalizer. After that, in data transmission mode, the data waiting for transmission is sent out at sender, and the received data without ISI. Therefore, the communication system can communicate normally. Fig. 1 is the schematic of equalizer training mode.

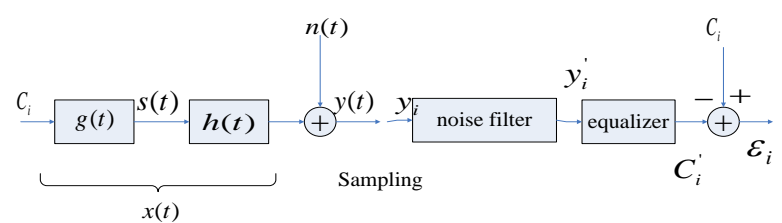

Fig.1.The schematic of equalizer training mode

In Fig.1, $C_{i}$ is the training sequence, $S(t)$ is the signal obtained by using pulse amplitude modulation (PAM), $h(t)$ is the channel's impulse response, $n(t)$ is noise, $y(t)$ is the received signal, $y_{i}$ is the discrete signal after sampling, $y_{i}^{\prime}$ is the discrete signal after filtering noise, $W_{i}$ is the I-th tap coefficient of the equalizer, $C_{i}^{\prime}$ is the estimated value of $C_{i}$ after equalization, $\varepsilon_{i}$ is the deviation between the transmitted signal and the estimated signal.

$$
\begin{aligned}
& S(t)=\sum_{i} C_{i} g(t-i T) \\
& x(t)=h(t) * g(t) \\
& y(t)=\sum_{i} C_{i} x(t-i T) \\
& y_{i}^{\prime}=\sum_{k=-\infty}^{\infty} x_{k} C_{i-k} \text { (Ignoring the noise) } \\
& C_{i}^{\prime}=\sum_{k=-\infty}^{\infty} W_{k} y_{i-k}^{\prime} \\
& \varepsilon_{i}=C_{i}-C_{i}^{\prime}
\end{aligned}
$$

\section{Zero forcing algorithm}

Zero forcing algorithm equalizer is the equalizer based on the criterion of minimum peak distortion, and constructing a tap matrix $\left\{W_{i}\right\}$ ensures that $\varepsilon_{i}=C_{i}-C_{i}^{\prime}=0$. Construction process of $\left\{W_{i}\right\}$ is as follows. $h_{i}$ is the whole system function after adding the equalizer.

$$
\begin{aligned}
& h^{\prime}(t)=x(t)^{*} e(t)=\sum_{k=-\infty}^{\infty} W_{k} x\left(t-k T_{b}\right) \\
& C_{i}^{\prime}=\sum_{k=-\infty}^{\infty} W_{k} y_{i-k}^{\prime}=\sum_{k=-\infty}^{\infty} W_{k} \sum_{m=-\infty}^{\infty} x_{i-k-m} C_{m}=\sum_{k=-\infty}^{\infty} \sum_{m=-\infty}^{\infty} W_{k} x_{i-k-m} C_{m}=\sum_{M=-\infty}^{\infty} h_{M}^{\prime} C_{i-M}=C_{i}(\mathrm{M}=\mathrm{i}-\mathrm{m}) \\
& h^{\prime}(t)=\sum_{k=-\infty}^{\infty} W_{k} x_{i-k}=\left\{\begin{array}{l}
0, i=0 \\
1, i \neq 0
\end{array}\right.
\end{aligned}
$$

If Eq.9 is established, the system satisfies no ISI. But in the practical application, an infinite number of taps is impossible. So it is necessary to consider reducing the number of taps, such as $2 \mathrm{~N}+1$. Assuming the training sequences is impulse response, and it becomes the equalizer's input signal $\left\{x_{k}\right\}$ after passing the channel. Then the zero forcing algorithm can be expressed as Eq.10.

$$
\left[\begin{array}{cccc}
x_{0} & & & x_{-2 N} \\
\vdots & \ddots & & \vdots \\
\vdots & & \ddots & \vdots \\
x_{2 N} & & & x_{0}
\end{array}\right]\left[\begin{array}{c}
W_{-N} \\
\vdots \\
W_{0} \\
\vdots \\
W_{N}
\end{array}\right]=\left[\begin{array}{c}
0 \\
\vdots \\
1 \\
\vdots \\
0
\end{array}\right]
$$




\section{Minimum mean square error algorithm}

MMSE algorithm equalizer is the equalizer based on minimum mean square error criterion. Adjusting the coefficient ensures that the mean square error between the desired signal $c_{\mathrm{i}}$ and the equalizer output signal $C_{i}^{\prime}$ become the minimum and meet $E\left\{\left|e_{i}\right|^{2}\right\} \rightarrow \min$. Construction process of $\left\{W_{i}\right\}$ is as follows.

$$
A(W)=E\left\{\left.e_{i}\right|^{2}\right\}=E\left\{\left|C_{i}-\sum_{k=-\infty}^{\infty} W_{k} y_{i-k}^{\prime}\right|^{2}\right\} \rightarrow \min
$$

In Eq.11, $A(W)$ is the error function. If there is a minimum value, the minimum value must be got on the pole. And the partial derivative of $A(W)$ is zero at this minimum value.

$$
\begin{aligned}
& \frac{\partial A}{\partial W_{k}}=E\left[\frac{\partial E\left\{\left|C_{i}-\sum_{k=-\infty}^{\infty} W_{k} y_{i-k}^{\prime}\right|^{2}\right\}}{\partial W_{k}}\right]=0 \\
& E\left[C_{i} y_{i-k}^{\prime}\right]-E\left[y_{i-k}^{\prime} \sum_{m=-N}^{N} W_{m} y_{i-m}^{\prime}\right]=E\left[C_{i} y_{i-k}^{\prime}\right]-\sum_{m=-N}^{N} W_{m} R_{y y}(k-m)=0 \\
& R_{c y}(k)=E\left[C_{i} y_{i-k}^{\prime}\right] \\
& R_{c y}(k)=\sum_{m=-N}^{N} W_{m} R_{y y}(k-m)
\end{aligned}
$$

Eq.14 is the cross correlation function of sending and receiving signal, and $R_{y y}(k)$ is the autocorrelation function of the received signal. The matrix representation is Eq.16. Finally, the error function can be calculated.

$$
\left[\begin{array}{cccc}
R_{y y}(0) & & & R_{y y}(-2 N) \\
\vdots & \ddots & & \vdots \\
\vdots & & \ddots & \vdots \\
R_{y y}(2 N) & & & R_{y y}(0)
\end{array}\right]\left[\begin{array}{c}
W_{-N} \\
\vdots \\
W_{0} \\
\vdots \\
W_{N}
\end{array}\right]=\left[\begin{array}{c}
R_{c y}(N) \\
\vdots \\
R_{c y}(0) \\
\vdots \\
R_{c y}(-N)
\end{array}\right] \Leftrightarrow W=R_{c y} R_{y y}{ }^{-1}
$$

\section{Simulation}

Experiment 1 (ZF equalization). Eq.10 is the formula for calculating the tap coefficient of ZF algorithm. In this simulation, using the function pinv calculates 5,17 and 31 tap equalizer's tap coefficients $C_{1}, C_{2}$ and $C$ respectively, and using the function symerr gets the BER under different SNR, their BER curves are presented.

In Fig.2, the conclusion can be obtained. In the ideal channel, the higher SNR is, the less influence of noise on the signal is, and the less BER is. So only a smaller increase of SNR can lead the error rate reduce quickly. While in the actual fading channel, the error case of lower SNR is very serious, so it's necessary to greatly enhance the transmission power to improve the SNR, to reduce bit error rate. The simulation results show that the SNR will be significantly improved after using equalizer, and BER could decrease rapidly with the increasing of SNR. What's more, the more taps there are, the better equalization effect will be obtained, and its curve is closer to the curve which have no ISI. Therefore, zero forcing equalizer can improve the quality of communication, the more number of taps there is, the better effect of equilibrium will be obtained. 
Experiment 2 (MMSE equalization). Eq.16 is the formula for calculating the tap coefficient of MMSE algorithm, equivalent to the simulation program in $W=R_{c y} R_{y y}{ }^{-1}$. In this simulation, using the function pinv calculates MMSE equalizer's tap coefficients $W_{-} m m s e=\operatorname{pinv}(R y y) * R c y^{-1}$, and using the function nErr_mmse(1,i) = size(find([ip- ipHat_mmse]),2) gets the number of errors, so nErr_mmse/ $\mathrm{N}$ is the BER via equalization, then their bit error rate curves are presented. In Fig.3, the conclusion can be obtained. In fading channel, the BER will be improved after using 7 taps of MMSE equalizer. The bigger the SNR is, the smaller the BER becomes. Therefore, MMSE equalizer can improve the quality of communication.

Experiment 3 (contrast). In Fig.4, the conclusion can be obtained. ISI seriously affect the accuracy of the received signal, but after using equalizer, the quality of communication can be improved, and the error rate can be reduced. What's more, the effect of MMSE equalizer is better than the ZF equalizer.

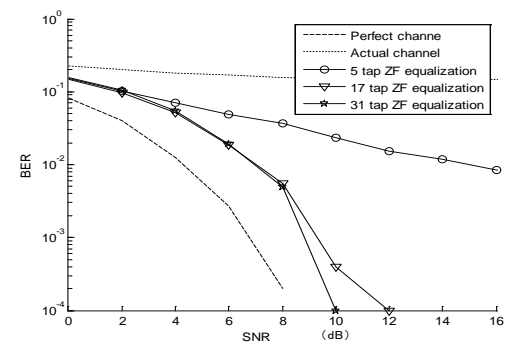

Fig.2. ZF equalization simulation

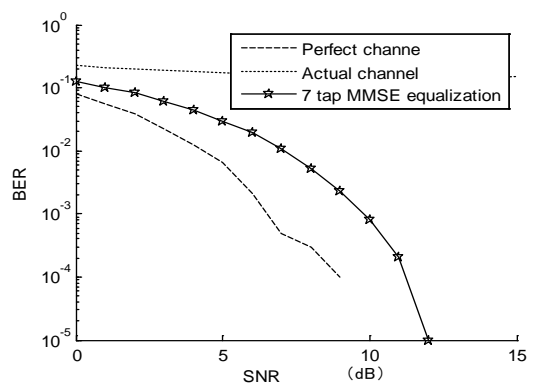

Fig.3. MMSE equalization simulation

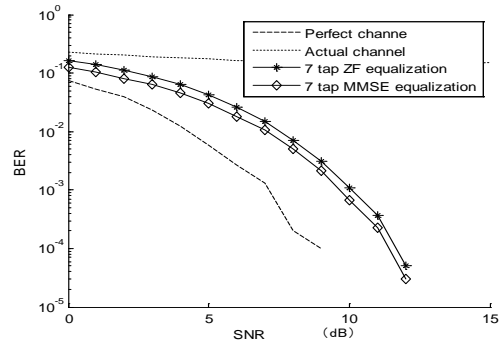

Fig.4.Comparison

\section{Conclusion}

In digital and information era, the communication system is taking on a major task. This requires the communication system expand constantly and have higher speed and reliability. As a very important technology in modern digital communication systems equalization technology can effectively eliminate the ISI caused by multipath channel, overcome the channel's non-ideal characteristics, and reduce signal distortion and error rate. So the equalization technology is indispensable. The conclusions are as follows. Firstly, the equalization technique can eliminate ISI and reduce the error rate. Secondly, the equalization effect is more obvious via using more equalizer taps. Thirdly, the effect of MMSE equalizer is better than the ZF equalizer.

\section{Acknowledgements}

I am grateful and honest appreciation to the teachers and students in Xi'an University of Science and Technology for their wised advices, help and encouragements throughout this study.

\section{References}

[1] Molisch A.F. Wireless communications [M]. Electronic Industry Press, 2008.

[2] Guihua Kang. The principle and application of MIMO wireless communication (In Chinese) [M]. Electronic Industry Press, 2009.

[3] Yongsheng Chen, Fang Gong, Yangyang Fang. Science technology magazine online, 2014.

[4] Xuefei Ma, Chunhui Zhao, Gang Qiao. Technical Acoustics, 2009, 28(4): 467-471.

[5] Rongtao Ma, Yuan Feng. science and technology information, 2008, $29: 52$.

[6] Siri Kors communication equipment Co. Ltd.. The realization of wireless communication based on MATLAB and FPGA (In Chinese) [M]. posts and telecom press, 2009, 6. 